\title{
ASYMPTOTIC BEHAVIOUR OF COMPOUND DISTRIBUTIONS \\ AND STOP-LOSS PREMIUMS
}

\author{
BY BJøRN SUNDT \\ University of Oslo/Eidgenössische Technische Hochschule (Zürich)
}

\begin{abstract}
The paper gives some asymptotic results for the compound distribution of aggregate claims when the claim number distribution is negative binomial. The case when the claim numbers are geometrically distributed, is treated separately.
\end{abstract}

\section{INTRODUCTION}

1A. Let $\tilde{x}_{1}, \tilde{x}_{2}, \ldots$ be independent identically distributed random variables (the independent severities) on $(0, \infty)$ with cumulative distribution $F$. Let $\tilde{n}$ be a random variable (the claim number), independent of the $\tilde{x}_{i}$ 's with distribution on the non-negative integers defined by

$$
p_{n}=\operatorname{Pr}(\tilde{n}=n)=\left(\begin{array}{c}
\alpha+n-1 \\
n
\end{array}\right) p^{n}(1-p)^{\alpha}, \quad(\alpha>0,0<p<1) .
$$

Let

$$
\tilde{s}=\left\{\begin{array}{ll}
\sum_{i=1}^{\tilde{n}} \tilde{x}_{i} & (\tilde{n}>0) \\
0 & (\tilde{n}=0)
\end{array}\right\}
$$

(the total aggregate claim amount). Then the cumulative distribution of $\tilde{s}$ is

$$
G(s)=\sum_{n=0}^{\infty} p_{n} F^{n^{*}}(s) \text {. }
$$

The idea of the present paper is to develop asymptotic expressions when $s \uparrow \infty$ for

(i) the tail

$$
H(s)=1-G(s)
$$

(ii) the stop-loss premium

$$
K(s)=\mathscr{C}(\max (\tilde{s}-s, 0))=\int_{s}^{\infty} H(x) d x ;
$$

The present research was supported by Association of Norwegian Insurance Companies and The Norwegian Research Council for Science and the Humanities. I am grateful to Hans Bühlmann, William S. Jewell, and André Dubey for useful discussions concerning the paper, and to Ragnar Norberg for suggesting several improvements. 
(iii) the density

$$
g(s)=\frac{d}{d s} G(s)
$$

or the point probability

$$
g_{s}=\operatorname{Pr}(\tilde{s}=s) .
$$

1B. We are going to use some notation and results from Feller (1971):

If $A$ and $B$ are functions, by the notation $A(s) \sim B(s)$ as $s$ tends to, say, $a$, we shall mean that the ratio $A(s) / B(s)$ tends to 1 as $s$ tends to $a$.

We shall call the severity distribution $F$ arithmetic if it is concentrated on the set $\{\lambda, 2 \lambda, 3 \lambda, \ldots\}$ for some $\lambda$, and we shall call the largest such $\lambda$ the span of the distribution. When we treat arithmetic distributions, we shall for convenience assume that the span is equal to one; general span-length is obtained by rescaling.

We shall say that a function $A$ is ultimately monotone if there exists a $y$ such that $A(x)$ is monotone for all $x>y$.

It is assumed that there exists a $\kappa$ satisfying

$$
\frac{1}{p}=\int_{(0, \infty)} e^{\kappa x} d F(x)
$$

and that

$$
\nu=p \int_{(0, \infty)} x e^{\kappa x} d F(x)
$$

is finite.

\section{GEOMETRICALLY DISTRIBUTED CLAIM NUMBER}

$2 \mathrm{~A}$. In the present section we are going to assume that $p_{n}$ satisfies (1) with $\alpha=1$, that is,

$$
p_{n}=p^{n}(1-p)
$$

Then the distribution $G$ satisfies the identity

$$
G(s)=1-p+p \int_{(0, s]} G(s-x) d F(x), \quad(s>0)
$$

as is seen by rewriting (2) as

$$
\begin{aligned}
G(s) & =\sum_{n=0}^{\infty} p^{n}(1-p) F^{n^{*}}(s) \\
& =1-p+p \sum_{n=0}^{\infty} p^{n}(1-p)\left(F^{n^{*}} * F\right)(x) \\
& =1-p+p(G * F)(s) .
\end{aligned}
$$


We see that (5) has the form of a renewal equation with defective distribution $p F$. This means that we can apply results from renewal theory. In subsections 2B-C we do this for the non-arithmetic case, in subsection 2D for the arithmetic case.

2B. Assume that $F$ is non-arithmetic. Then by formulae (6.7) and (6.16) of Chapter XI in Feller (1971) we get

$$
H(s) \sim \frac{1-p}{\kappa \nu} e^{-\kappa s}, \quad(s \uparrow \infty) .
$$

From this we can also easily obtain an asymptotic expression for the stop-loss premium $K(s)$.

THEOREM 1. The stop-loss premium $K(s)$ satisfies

$$
K(s) \sim \frac{1-p}{\kappa^{2} \nu} e^{-\kappa s}, \quad(s \uparrow \infty) .
$$

Proof. By using L'Hôpital's rule we get

$$
\lim _{s \uparrow \infty} \frac{K(s)}{e^{-\kappa s}}=\lim _{s \uparrow \infty} \frac{-H(s)}{-\kappa e^{-\kappa s}}=\frac{1-p}{\kappa^{2} \nu}
$$

which proves the theorem.

Q.E.D.

2C. If $F$ has a density $f$, then $G$ has an atom

$$
G(0)=p_{0}
$$

at zero, and for $s>0$ a density

$$
g(s)=\sum_{n=1}^{\infty} p_{n} f^{n^{*}}(s)
$$

THEOREM 2. The density $g(s)$ satisfies

$$
g(s) \sim \frac{1-p}{\nu} e^{-\kappa s}, \quad(s \uparrow \infty) .
$$

Proof. We use L'Hôpital's rule:

$$
\frac{1-p}{\kappa \nu}=\lim _{s \uparrow \infty} \frac{H(s)}{e^{-\kappa s}}=\lim _{s \uparrow \infty} \frac{-g(s)}{-\kappa e^{-\kappa s}}=\frac{1}{\kappa} \lim _{s \uparrow \infty} \frac{g(s)}{e^{-\kappa s}} .
$$

From this follows the theorem.

Q.E.D.

2D. In this subsection we shall assume that the distribution $F$ is arithmetic with unit span, and we introduce

$$
f_{k}=\operatorname{Pr}\left(\tilde{x}_{i}=k\right), \quad(k=1,2, \ldots) .
$$


Then

$$
F(x)=\sum_{i=1}^{[x]} f_{i}, \quad(x \geqslant 0)
$$

THEOREM 3. When s goes to infinity through the integers, we have

$$
\begin{aligned}
\text { (i) } K(s) & \sim \frac{(1-p) e^{\kappa}}{\nu\left(e^{\kappa}-1\right)^{2}} e^{-\kappa s} \\
\text { (ii) } H(s) & \sim \frac{1-p}{\nu\left(e^{\kappa}-1\right)} e^{-\kappa s} \\
\text { (iii) } g_{s} & \sim \frac{1-p}{\nu e^{\kappa}} e^{-\kappa s} .
\end{aligned}
$$

Proof. For the whole proof $s$ will always denote a non-negative integer.

(i) In the present case (5) becomes

$$
G(s)=1-p+p \sum_{i=1}^{s} G(s-i) f_{i} .
$$

As $G(s-i)=0$ for $i>s$, we may extend the sum to infinity,

$$
G(s)=1-p+p \sum_{i=1}^{\infty} G(s-i) f_{i} .
$$

Introduction of $H(s)=1-G(s)$ gives

$$
H(s)=p \sum_{i=1}^{\infty} H(s-i) f_{i}
$$

We get

$$
\begin{aligned}
K(s) & =\sum_{x=s}^{\infty} H(x)=\sum_{x=s}^{\infty} p \sum_{i=1}^{\infty} H(x-i) f_{i} \\
& =p \sum_{i=1}^{\infty}\left(\sum_{y=s-i}^{\infty} H(y)\right) f_{i},
\end{aligned}
$$

and thus

$$
K(s)=p \sum_{i=1}^{\infty} K(s-i) f_{i}
$$

By using

$$
K(s-i)=\mathscr{E}(\tilde{s})+i-s, \quad(i=s+1, s+2, \ldots)
$$

we obtain

$$
K(s)=p \sum_{i=s+1}^{\infty}(\mathscr{E}(\tilde{s})+i-s) f_{i}+\sum_{i=1}^{s} K(s-i) p f_{i}
$$


Multiplying this equation by $e^{\kappa s}$ and introducing

$$
f_{i}^{*}=p f_{i} e^{\kappa i}
$$

and

$$
K^{*}(s)=K(s) e^{\kappa s}
$$

give

$$
K^{*}(s)=p e^{\kappa s} \sum_{i=s+1}^{\infty}(\mathscr{E}(\tilde{s})+i-s) f_{i}+\sum_{i=1}^{s} K^{*}(s-i) f_{i}^{*}
$$

Considered as point probabilities $f_{i}^{*}, f_{2}^{*}, \ldots$ defines a proper probability distribution because of (3). Hence (10) is a proper renewal equation, and the renewal theorem (Karlin and Taylor, 1975, p. 81) gives

$$
\lim _{s \uparrow \infty} K^{*}(s)=\frac{1}{\nu} \sum_{j=0}^{\infty} p e^{\kappa j} \sum_{i=j+1}^{\infty}(\mathscr{E}(\tilde{s})+i-j) f_{i} .
$$

In the following development we use that

$$
\mathscr{E}(\tilde{s})=\mathscr{E}(\tilde{n}) \mathscr{E}\left(\tilde{x}_{1}\right)=\frac{p}{1-p} \mathscr{E}\left(\tilde{x}_{1}\right)
$$

We have

$$
\begin{aligned}
\nu \lim _{s \uparrow \infty} K^{*}(s) & =\sum_{i=0}^{\infty} p e^{\kappa j} \sum_{i=j}^{\infty}(\mathscr{E}(\tilde{s})+i+1-j) f_{i+1} \\
& =p \sum_{i=0}^{\infty} f_{i+1} \sum_{j=0}^{i}(\mathscr{E}(\tilde{s})+i+1-j) e^{\kappa i} \\
& =p \sum_{i=0}^{\infty} f_{i+1}\left[\frac{e^{\kappa(i+1)}-1}{e^{\kappa}-1} \mathscr{E}(\tilde{s})+\sum_{j=0}^{i} \sum_{k=j}^{i} e^{\kappa i}\right] \\
& =p\left[\frac{\mathscr{E}(\tilde{s})}{e^{\kappa}-1}\left(\frac{1}{p}-1\right)+\sum_{i=0}^{\infty} f_{i+1} \sum_{k=0}^{i} \sum_{j=0}^{k} e^{\kappa i}\right] \\
& =p\left[\frac{\mathscr{E}(\tilde{x})}{e^{\kappa}-1}+\sum_{i=0}^{\infty} f_{i+1} \sum_{k=0}^{i} \frac{e^{\kappa(k+1)}-1}{e^{\kappa}-1}\right] \\
& =\frac{p}{e^{\kappa}-1}\left[\mathscr{E}(\tilde{x})+\sum_{i=0}^{\infty} f_{i+1} \sum_{k=0}^{i}\left(e^{\kappa(k+1)}-1\right)\right] \\
& =\frac{p}{e^{\kappa}-1}\left[\mathscr{E}(\tilde{x})+\sum_{i=0}^{\infty} f_{i+1}\left(\frac{e^{\kappa(i+2)}-e^{\kappa}}{e^{\kappa}-1}-(i+1)\right)\right] \\
& =\frac{p}{e^{\kappa}-1}\left[\mathscr{E}(\tilde{x})+\frac{e^{\kappa}}{e^{\kappa}-1}\left(\frac{1}{p}-1\right)-\mathscr{E}(\tilde{x})\right]=\frac{(1-p) e^{\kappa}}{\left(e^{\kappa}-1\right)^{2}} .
\end{aligned}
$$

From this follows (i). 
(ii) As

we get

$$
H(s)=\boldsymbol{K}(s)-\boldsymbol{K}(s+1),
$$

$$
\begin{aligned}
\lim _{s \uparrow \infty} H(s) e^{\kappa s} & =\lim _{s \uparrow \infty} K(s) e^{\kappa s}-e^{-\kappa} \lim _{s \uparrow \infty} K(s+1) e^{\kappa(s+1)} \\
& =\left(1-e^{-\kappa}\right) \lim _{s \uparrow \infty} K(s) e^{\kappa s},
\end{aligned}
$$

which proves (ii).

(iii) As

$$
g_{s}=H(s)-H(s+1),
$$

the proof of (iii) goes as the proof of (ii).

This completes the proof of Theorem 3 .

Q.E.D.

\section{NEGATIVE BINOMIALLY DISTRIBUTED CLAIM NUMBER}

3A. We shall now drop the restriction $\alpha=1$ in (1). Then we have the following theorems:

THEOREM 4. If

$$
R(s)=e^{\kappa s} H(s)
$$

is ultimately monotone, then

$$
H(s) \sim \frac{1}{\kappa \Gamma(\alpha)}\left(\frac{1-p}{\nu}\right)^{\alpha} s^{\alpha-1} e^{-\kappa s}, \quad(s \uparrow \infty) .
$$

ProOF. Let

$$
\begin{array}{ll}
\phi(t)=\int_{(0, \infty)} e^{-t x} d F(x), & (t \geqslant-\kappa) \\
\sigma(t)=\int_{[0, \infty)} e^{-t s} d G(s)=\left(\frac{1-p}{1-p \phi(t)}\right)^{\alpha}, & (t>-\kappa) \\
\omega(t)=\int_{[0, \infty)} e^{-t s} R(s) d s=\frac{1-\sigma(t-\kappa)}{t-\kappa}, & (0<t<\kappa) .
\end{array}
$$

We want to show that

$$
R(s) \sim \frac{1}{\kappa \Gamma(\alpha)}\left(\frac{1-p}{\nu}\right)^{\alpha} s^{\alpha-1} . \quad(s \uparrow \infty)
$$

By Theorem 4 on p. 446 in Feller (1971) this is equivalent to

$$
\omega(t) \sim \frac{1}{\kappa}\left(\frac{1-p}{\nu t}\right)^{\alpha}, \quad(t \downarrow 0) .
$$


Let

$$
\psi(t)=\kappa\left(\frac{\nu t}{1-p}\right)^{\alpha} \omega(t)
$$

We have to show that

$$
\lim _{t \downarrow 0} \psi(t)=1
$$

We have

$$
\begin{aligned}
\lim _{t \downarrow 0} \psi(t) & =\lim _{t \downarrow 0} \frac{1-\sigma(t-\kappa)}{t-\kappa}\left(\frac{\nu t}{1-p}\right)^{\alpha} \kappa \\
& =\lim _{t \downarrow 0} \sigma(t-\kappa)\left(\frac{\nu t}{1-p}\right)^{\alpha} \\
& =\lim _{t \downarrow 0}\left(\frac{\nu t}{1-p \phi(t-\kappa)}\right)^{\alpha} .
\end{aligned}
$$

As, by L'Hôpital's rule,

$$
\lim _{t \downarrow 0} \frac{\nu t}{1-p \phi(t-\kappa)}=\lim _{t \downarrow 0} \frac{\nu}{-p \phi^{\prime}(t-\kappa)}=\frac{\nu}{-p \phi^{\prime}(-\kappa)}=1,
$$

(13) holds, and hence the theorem is proved.

Q.E.D.

THEOREM 5. If (11) holds, then

$$
K(s) \sim \frac{1}{\kappa^{2} \Gamma(\alpha)}\left(\frac{1-p}{\nu}\right)^{\alpha} s^{\alpha-1} e^{-\kappa s}, \quad(s \uparrow \infty) .
$$

Proof. L'Hôpital's rule gives

$$
\begin{aligned}
\lim _{s \uparrow \infty} \frac{K(s)}{s^{\alpha-1} e^{-\kappa s}} & =\lim _{s \uparrow \infty} \frac{-H(s)}{-\kappa s^{\alpha-1} e^{-\kappa s}+(\alpha-1) s^{\alpha-2} e^{-\kappa s}} \\
& =\lim _{s \uparrow \infty} \frac{\kappa s}{\kappa s-\alpha+1} \frac{H(s)}{\kappa s^{\alpha-1} e^{-\kappa s}} \\
& =\lim _{s \uparrow \infty} \frac{H(s)}{\kappa s^{\alpha-1} e^{-\kappa s}}=\frac{1}{\kappa{ }^{2} \Gamma(\alpha)}\left(\frac{1-p}{\nu}\right)^{\alpha},
\end{aligned}
$$

which proves the theorem.

Q.E.D.

3B. If $F$ has a density $f$, then $G$ has an atom $p_{0}$ at zero, and for $s>0$ a density $g(s)$ given by (7). Then we have the following theorem.

THEOREM 6. If (11) holds, the density $g(s)$ satisfies

$$
g(s) \sim \frac{1}{\Gamma(\alpha)}\left(\frac{1-p}{\nu}\right)^{\alpha} s^{\alpha-1} e^{-\kappa s}, \quad(s \uparrow \infty) .
$$


ProOF, L'Hôpital's rule gives

$$
\begin{aligned}
\frac{1}{\kappa \Gamma(\alpha)}\left(\frac{1-p}{\nu}\right)^{\alpha} & =\lim _{s \uparrow \infty} \frac{H(s)}{s^{\alpha-1} e^{-\kappa s}}=\lim _{s \uparrow \infty} \frac{-g(s)}{-\kappa s^{\alpha-1} e^{-\kappa s}+(\alpha-1) s^{\alpha-2} e^{-\kappa s}} \\
& =\lim _{s \uparrow \infty} \frac{\kappa s}{\kappa s-\alpha+1} \frac{g(s)}{\kappa s^{\alpha-1} e^{-\kappa s}}=\lim _{s \uparrow \infty} \frac{g(s)}{\kappa s^{\alpha-1} e^{-\kappa s}},
\end{aligned}
$$

and this proves the theorem.

Q.E.D.

3C. The assumption that $R(s)$ is ultimately monotone, is awkward, as it seems very difficult to show that it is satisfied. As, when the condition holds,

$$
R(s) \sim R^{*}(s), \quad(s \uparrow \infty)
$$

with

$$
R^{*}(s)=\frac{1}{\kappa \Gamma(\alpha)}\left(\frac{1-p}{\nu}\right)^{\alpha} s^{\alpha-1}
$$

which is monotonely increasing to infinity for $\alpha>1$, constant for $\alpha=1$, and monotonely decreasing to zero for $\alpha<1$, the condition must mean that $R(s)$ is ultimately monotonely increasing for $\alpha>1$ and ultimately monotonely decreasing for $\alpha<1$; for $\alpha=1$ we cannot say whether the ultimate monotony is increasing or decreasing, but in that case it does not matter as we then have the theorems of Section 2.

In the arithmetic case the assumption of ultimate monotony of $R(s)$ does not hold as $R(s)$ then increases continuously when $s$ is a non-integer and decreases in jumps at integers. But in this case Theorems 4 and 5 cannot hold as for $\alpha=1$ (9) and (8) contradict (11) and (14).

If $F$ has a density $f$, the following lemma gives a condition equivalent to ultimate monotony of $R(s)$ when $\alpha \neq 1$.

LeMma 1. Assume that $F$ has a density $f$ and that $\alpha \neq 1$. Then $R(s)$ is ultimately monotone if and only if there exists an $s_{\alpha}$ such that for all $s>s_{\alpha}$

$$
\begin{array}{ll}
\frac{g(s)}{H(s)}<\kappa, & (\alpha>1) \\
\frac{g(s)}{H(s)}>\kappa, & (\alpha<1) .
\end{array}
$$

ProOF. For $s>0$

$$
\frac{d R}{d s}=e^{\kappa s}(\kappa H(s)-g(s)),
$$

and hence

$$
\frac{d R}{d s} \gtrless 0 \Leftrightarrow \frac{g(s)}{H(s)} \lessgtr \kappa .
$$


But because of the ultimate monotony of $R(s)$ there must exist an $s_{\alpha}$ such that for all $s>s_{\alpha}, d R / d s \gtrless 0$ as $\alpha \gtrless 1$. This proves the lemma.

Q.E.D.

The author believes that the assumption of ultimate monotony of $R(s)$ in Theorem 4 may be replaced by the assumption that the distribution $F$ is non-arithmetic, but has not been able to prove this result. An indication that the result holds, is that it holds in the special case $\alpha=1$ as shown in subsection 2B. Another indication is given by the following example.

EXAMPLE. Let the severity distribution $F$ be defined by the density

$$
f(x)=\beta e^{-\beta x}, \quad(x>0, \beta>0) .
$$

Then the Laplace transform of $F$ is

$$
\phi(t ; \beta)=\frac{\beta}{\beta+t},
$$

and from (12) follows that the Laplace transform of $G$ is

$$
\sigma(t)=(1-p+p \phi(t ; \beta(1-p)))^{\alpha} .
$$

By expanding we get

$$
\sigma(t)=\sum_{i=0}^{\infty}\left(\begin{array}{c}
\alpha \\
i
\end{array}\right)(1-p)^{\alpha-i} p^{i} \phi(t ; \beta(1-p))^{i}
$$

As $\phi(t ; \beta(1-p))^{i}$ is the Laplace transform of the distribution $\Gamma(s ; i, \beta(1-p))$ defined by

$$
\Gamma(s ; a, b)=\frac{b^{a}}{\Gamma(a)} \int_{0}^{s} r^{a-1} e^{-b r} d r, \quad(s, a, b>0)
$$

we get

$$
H(s)=\sum_{i=0}^{\infty}\left(\begin{array}{c}
\alpha \\
i
\end{array}\right)(1-p)^{\alpha-i} p^{i}(1-\Gamma(s ; i, \beta(1-p)) .
$$

Assume that $\alpha$ is an integer. Then

$$
H(s)=\sum_{i=0}^{\alpha}\left(\begin{array}{c}
\alpha \\
i
\end{array}\right)(1-p)^{\alpha-i} p^{i}(1-\Gamma(s ; i, \beta(1-p)) .
$$

As by L'Hôpital's rule

$$
\lim _{s \uparrow \infty} \frac{1-\Gamma(s ; i, \beta(1-p))}{s^{\alpha-1} e^{-\beta(1-p) s}}= \begin{cases}0, & (i<\alpha) \\ \frac{[\beta(1-p)]^{\alpha-1}}{\Gamma(\alpha)}, & (i=\alpha)\end{cases}
$$

we get

$$
H(s) \sim \frac{1}{\beta(1-p) \Gamma(\alpha)}(\beta(1-p) p)^{\alpha} s^{\alpha-1} e^{-\beta(1-p) s}, \quad(s \uparrow \infty) .
$$


From (3) and (15) we get

$$
\frac{1}{p}=\frac{\beta}{\beta-\kappa}
$$

that is,

$$
\kappa=\beta(1-p),
$$

and inserting this in (4) gives

$$
\nu=\frac{1}{\beta p}
$$

By inserting (17) and (18) in (16) we arrive at (11), which then holds also in the case when $\alpha$ is an integer and $F$ is the exponential distribution.

\section{REFERENCES}

Feller, W. (1971). An Introduction to Probability Theory and its Applications. Vol. II (2nd. ed.): Wiley, New York.

Karlin, S. and TAYlor, H. M. (1975). A First Course in Stochastic Processes. Academic Press: New York. 